\title{
Chlamydia screening: which sample for which technique?
}

\section{Symposium}

\author{
Angelika Stary
}

Outpatients' Centre for Diagnosis of Infectious VeneroDermatological Diseases, A-1210 Vienna, Franz JonasPlatz 8-2-3, Austria A Stary

Accepted for publication 3 March 1997
Chlamydia trachomatis is considered the most common bacteria causing genital infections in the industrialised world. In women, the infection may lead to serious complications such as pelvic inflammatory disease (PID) with consequences including infertility, ectopic pregnancy, and chronic pelvic pain. It has been shown that screening of women at risk for STD can reduce half of the cases of PID. ${ }^{1}$

Within the past decade different nonculture tests have become commercially available to enable more efficient diagnostic and screening programmes even in large population groups. ${ }^{2}$ Owing to the high sensitivity of some of these tests invasive specimens from the endocervical canal and urethra can now be replaced by non-invasive specimens such as urine and vulval samples.

Which tests are currently available for chlamydial diagnosis?

Beside cell culture, a variety of commercially available laboratory tests such as antigen detection tests, nucleic acid hybridisation assays, and nucleic acid amplification techniques can now be used for the diagnosis of chlamydial infections in women and men on different sample types.

\section{CELL CULTURE}

For years, culture of the organism was the only recommended technique for chlamydial diagnosis and has been regarded as the "gold standard" method to be compared with all other techniques. Cell culture has the benefit of an excellent specificity while the sensitivity is now recognised to be less than optimal. The sensitivity of detection of $C$ trachomatis by cell culture even with experienced laboratory workers is probably not higher than up to $70 \%$ in females and males depending on the laboratory procedures and on the clinical status of the patient and the number of viable organisms in the specimen. ${ }^{3}$ Cell culture procedures differ in research centres and are not standardised. ${ }^{4}$ The reliability of diagnosis by cell culture depends not only on the expertise of the cell culture technique itself. A substantial variation was noted in specimen collection, transport conditions and times, culture procedures and identification of positive cultures by different staining methods and by performing subcultures in case of a negative result. Pitfalls, other than a low sensitivity of culture, are a bacterial overgrowth and cytopathic effect especially for cervical specimens.

\section{ANTIGEN DETECTION}

Antigen detection tests, such as enzyme immunoassays (EIA) and direct immunofluorescence assays (DFA), use monoclonal or polyclonal antibodies against the major outer membrane protein (MOMP) or the lipopolysaccharide (LPS) of $C$ trachomatis. At the present, the most commonly used tests for chlamydial diagnosis are EIAs. The sensitivity ranges from less than $70 \%$ up to $100 \%$ when compared with cell culture and is dependent on the level of organisms present in the specimen and the ability of the test to detect even a low load of elementary bodies (EBs). Because of the high number of EBs in symptomatic patients a median sensitivity of up to $92 \%$ can be achieved in this patient group. ${ }^{5}$ The ranges of sensitivity of antigen tests in different studies are not only influenced by the quality of the test itself but also by the quality of culture or other comparative tests used for the calculation. ${ }^{6}$ Including amplification assays as gold standard the detection rate of EIAs is decreased to $40-80 \%$. The test performance of EIAs is simple, for some are already fully automatic and reading the results is objective, allowing a high test capacity. Because of cross reactivities with other bacteria, positive results obtained by the EIA must be confirmed by the DFA on the sediment after centrifugation of the test tube or by an additional blocking test of the residual specimen. For chlamydial diagnosis by the DFA, transport and storage conditions are unimportant, the processing of specimens is simple, and both the sensitivity and specificity of the DFA depend on the experience of the observer to detect small numbers of EBs and to discriminate between specific and non-specific staining. This technique is the only one which gives an insight into the quality of specimen collection by the presence of a sufficient number of cells on the slide, but it has the disadvantage of a low capacity of the number of specimens to be examined, depending on the observer's expertise. The DFA is often included in evaluation studies for discrepant analysis of culture negative and non-culture test positive results.

\section{NUCLEIC ACID HYBRIDISATION TESTS}

Nucleic acid probe assays have been shown to be effective alternatives to EIAs for screening for $C$ trachomatis. ${ }^{7-9}$ The commercially available PACE 2 assay (Gen-Probe) has been recorded to be as sensitive as EIAs or culture. ${ }^{79}$ The use of the method has become widespread with the advantage of a high test 
capacity. The storage and transport conditions are similar to the EIAs, the procession of specimens is less technical than cell culture, and reading of the test is simple and objective. There is a grey zone of borderline or low positive results which have to be confirmed by the probe competition assay (PCA) to differentiate between false and true positive cases and to increase both the sensitivity and specificity of the PACE 2. Since an RNA amplification step has recently been developed to be included in this test, the PACE 2 test will be replaced by this amplification assay in the near future.

AMPLIFICATION ASSAYS

It was recently demonstrated that nucleic acid amplification by the ligase chain reaction (LCR, Abbott Laboratories, Abbott Park, IL, USA) and the polymerase chain reaction (PCR) represents a highly sensitive and specific approach for the detection of $C$ trachomatis in genital specimens of men and women when compared with cell culture..$^{10-11}$ Both techniques are based on the amplification of plasmid DNA specific for the organism.

For LCR, a co-amplification for the simultaneous detection of Neisseria gonorrhoeae from the same specimen has been developed and is already commercially available, although processing is done separately in the laboratory for both bacteria. The most recent development of amplification tests is the Gen-Probe amplified Chlamydia trachomatis assay which is a transcription mediated amplification (TMA) with the ribosomal RNA as the target to be amplified and detected. The TMA uses two primers, one containing a promoter sequence for the RNA polymerase and a second primer binding to a DNA copy. One of the two enzymes, the reverse transcriptase creates a DNA copy of the target rRNA by extension from the 3' end of the promoter primer. The second enzyme, the RNA polymerase, recognises the promoter sequence in the DNA template and initiates transcription. Each of the DNA templates can make 100-1000 copies of RNA amplicon, resulting in a production of 10 billion amplicons in less than one hour. Detection of these amplicons occurs by the same hybridisation protection assay (HPA) which utilises a highly sensitive chemiluminescent signal as in the PACE 2 assay. One of the advantages of the TMA is the high number of rRNAs in each infected cell. Furthermore, the test procedure is simple to perform, the TMA is an isothermal process and does not require a thermocycler for the amplification step.

For amplification tests transport and storage conditions are less critical when compared with culture. Transport media are usually provided by the manufacturer. Although processing of the test requires precautions against contamination, the performance is already partly automated, and reading is objective and simple. The sensitivity of amplification assays is considerably increased compared with nonamplification procedures including cell culture, and the discrepancy between other tests is especially high for specimens with a low number of chlamydial particles, as may occur in asymptomatic people. ${ }^{12}$

\section{Which samples can be used for which} test?

Genital infections caused by $C$ trachomatis are due to an infection of the columnar or transi-? tional epithelium of the urethra and the endo-J cervix, with extension to the upper genital? tract such as the epididymis, endometrium,? salpinx, and the peritoneum. Rarely, the rectum can also harbour the organism. For chlamydial diagnosis, invasive samples from등 the site of infection (genital samples) with a high number of viable organisms as well as $\frac{\Phi}{\alpha}$ non-invasive samples (urine and vulval sam-® ples) contaminated with EBs may be used.

\section{INVASIVE SAMPLES}

For the diagnosis of genital chlamydial infec-? tions by cultivation of the organism only genital $\omega$ specimens from the site of infection with a sufficient number of viable EBs are recommended. Specimen collection with as many cells as possible from the urethra or the endo- $\rightarrow$ cervical canal, and optimal transport and stor-을 age condition for the specimens to preserve viable organisms for cultivation are important issues.

Of the antigen detection tests, the DFA tests have been used predominantly for the detection of infectious organisms in genital and ocular smears. Reading the test becomes $\mathbb{Q}$ especially tedious when a low number of fewer than 10 EBs are present in the smear, as can happen in asymptomatic people. It may become difficult to discriminate between spe-? cific and non-specific staining if fewer than five EBs are present.

EIAs have been recommended primarily for the examination of genital samples of men and $\delta$ women with a sufficiently high number of EBs being detected in the specimen. Sensitivity val- 윽 ues vary for different EIAs, depending on their $\frac{D}{0}$ capacity to detect even small numbers of EBs. The specificity of EIAs is influenced by the $\sigma$ presence of other Gram negative bacteria $N$ especially in pharyngeal, urine, or ocular $\mathbb{E}^{2}$ swabs because of cross reactivity.

The PACE 2 test is recommended for use only for genital samples.

The evaluation of amplification tests started with comparison studies of genital samples using either cervical or urethral swabs from $\mathbb{D}$ men and women. Because of the high sensitiv- $\mathbb{D}$ ity of amplifying tests the results are less dependent on the quality of specimens and an inadequately collected or transported specimen with a low number of infectious agents can be dealt with.

\section{NON-INVASIVE SAMPLES}

In contrast with genital samples, non-invasive specimens such as urine and introital smears from the vulval region are easy to collect, giving access to a large population group, and so are 
important for screening adolescents with a high prevalence of asymptomatic chlamydial infections. Indeed, since in some women chlamydia can only be isolated from urethral samples, these tests may have higher detection rates through contamination from urethral and cervical specimens than samples taken from the cervix alone.

Since most of the organisms contaminating urine are no longer viable, urine as a non-invasive specimen is not an appropriate sample for chlamydial diagnosis by cell culture, as it gives a low sensitivity of up to $30 \%$ from urine samples. ${ }^{13}$ Furthermore, vulval smears obtained from the introitus vulvae should not be used to perform cell culture. In a comparative study using vulval specimens in addition to urine and cervical swabs the sensitivity of culture on vulval swabs was only $22 \cdot 2 \%$, similar to the data for urine. ${ }^{14}$

Antigen detection tests were not only evaluated for invasive but also for non-invasive samples, especially in men, suggesting a role of urine as screening tool. ${ }^{15-18}$ Chlamydial diagnosis on the deposit of centrifuged first void urine (FVU) has been performed with different EIAs mostly in men. The first results of urine testing looked promising when compared with culture, with a sensitivity of about $80 \%$ to $85 \%$ for the Chlamydiazyme and the EIA Baxter Bartels on male urine sediment in asymptomatic and symptomatic men. ${ }^{17} 18$ The problem at that time was that calculations were performed with culture as the gold standard for the specificity as well as sensitivity of EIAs on urine sediment. These enthusiastic first data reported in some studies had to be revised by the newly introduced amplifying techniques, which have shown a poor sensitivity for culture as well as for the EIA on urine especially in asymptomatic individuals, with a sensitivity not higher than $40 \% .{ }^{19}$

Although the diagnosis of EBs in the urine sediment by the DFA gave a better result for sensitivity and specificity than did EIAs, the disadvantage of the DFA on urine sediment is the labour intensive procedure and dependence on the expertise of the observer. Thus, it is not suitable for the performance of screening programmes in a larger population group.

Amplifying assays are highly effective in identifying genital chlamydial infections in men and in women not only by testing invasive samples but also by using FVU as a non-invasive specimen. ${ }^{1120-22}$ Automated LCR assay on FVU of about 2000 women detected up to $30 \%$ more infected women than did endocervical swab culture. ${ }^{21}$ So far, the LCR assay is highly effective for the detection of $C$ trachomatis even in urine of females with or without signs or symptoms of a genital chlamydial infection. Similar to LCR, testing urine sediment with the commercially available PCR Amplicor (Roche Diagnostic Systems, Branchburg, NJ, USA) performs with a higher sensitivity when compared with urethral and cervical culture. ${ }^{1123}$ In a study on 415 women, culture detected $61.2 \%$ of infected women compared with $87 \cdot 8 \%$ for the commercial PCR assay Amplicor on urine. ${ }^{11}$ Using the
Amplicor for testing urine in 713 men the sensitivity of culture on urethral specimens was only $50.7 \%$ and the resolved sensitivity of Amplicor on urine was $88 \%$, confirming that this technique provides a non-invasive diagnostic assay for routine screening for chlamydial infection.

Since in asymptomatic individuals only a small amount of infectious agents can be expected, detection of micro-organisms requires the use of nucleic acid amplification. This was shown in a recently performed comparative study in 705 asymptomatic male recruits. ${ }^{19} \mathrm{We}$ compared the performance of two different amplification assays, the LCR and PCR, with that of the EIA and FVU. The results confirm that both amplification methods were similarly sensitive $(93 \cdot 1 \%)$ using frozen urine of asymptomatic young men, which the EIA performed with a poor sensitivity of $37.9 \%$ and cannot be recommended as a suitable alternative test for urine, especially of asymptomatic men.

Recently, we investigated whether vulval specimens as an alternative non-invasive specimen in women may also be suitable for chlamydial diagnosis. ${ }^{14}$ In this study LCR, culture, and EIA were tested on different specimens from various sampling sites in women, including vulval smears and FVU as well as swabs from the endocervical canal and the urethra. The principal question was whether the vulval region may act as an alternative noninvasive sampling site which could substitute FVU for the detection of $C$ trachomatis in women. Whereas LCR showed a high sensitivity for all specimen types $(85 \cdot 2 \%$ for vulval, urine, and endocervical specimens, each), the sensitivity of culture and EIA was high only with endocervical swabs $(74 \cdot 1 \%$ and $70.4 \%$, respectively), and low for vulval specimens $(22 \cdot 2 \%$ and $40 \cdot 7 \%$ ). The high sensitivity for vulval and urine specimens using LCR was confirmed by a further study in 312 women, and seems not to be influenced by either the viability of the organism or by the low number of infectious particles. It can be explained as a result of the ability of this technology to amplify even a small amount of chlamydial DNA. Furthermore, it confirms the presence of EBs in the vulval region contaminated from the cervical and urethral region, similar to urine swabs. The low sensitivity of vulval swabs by culture is most probably the result of the low number of viable organisms as is the case with urine samples. The low sensitivity of the EIA when testing vulval swabs may be due to the low number of EBs in the specimens, below the detection limit of this technique. Vulval specimens as with FVU, seem not to be suitable for chlamydial diagnosis by culture or by EIA but only by the more sensitive amplification tests. The poor performance of antigen detection was also demonstrated on vaginal specimens by the Abbott Testpack assays on vaginal tampons. ${ }^{24}$ Similar to urine results, the detection rate of the Abbott Testpack assays on vaginal tampons was lower when compared with cervical swabs or culture because of a smaller amount of antigen in the vaginal fluid. 
In a comparative study performed in patients attending the outpatients' centre for STD diagnosis in Vienna, the TMA for $C$ trachomatis was compared with cell culture and with the LCR assay on endocervical, vulval, and urine samples for women, and urethral and urine samples for men. ${ }^{25}$ Both amplifying assays, the TMA and LCR, performed with a high sensitivity for all samples, detecting more infected individuals by testing urine and vulval specimens compared with culture on genital specimens. The concordance of TMA and LCR assays was high. In a recently published comparison study the results of urine tested by TMA were compared with those obtained by the AmplicorPCR and with culture. ${ }^{26}$ Both amplifying methods performed with a sensitivity of more than $90 \%$ on urine. The results presume that the TMA can be supposed to be another amplifying method for chlamydial diagnosis on non-invasive specimens.

A new century of chlamydial diagnosis has started with the possibility to amplify and detect even small amounts of the chlamydial DNA or RNA in non-invasive specimens and with the opportunity of screening a large percentage of the population at risk by easy sample collection. The results indicate that in addition to FVU vulval specimens also seem to be suitable as non-invasive samples for the highly sensitive amplifying assays LCR, PCR, and TMA, and may be used as an alternative to genital samples for screening high risk populations. Testing vulval specimens may replace the more time consuming urine testing which necessitates a centrifugation step in the laboratory. In addition, the sampling may be performed by the women themselves. Furthermore, it is essential that to evaluate the true sensitivities and specificities of new tests an amplifying assay must be included not only for discrepant analysis but to test all positive and negative samples to determine the reliability of a new test for all infected and noninfected individuals.

1 Scholes D, Stergachis A, Heidrich FE, Andrilla H, Holmes KK, Stamm WE. Prevention of pelvic inflammatory disease by screening for cervical chlamydial infection. $N$ Engl F Med 1996;344:1362-6.

2 Schachter J, Stamm WE, Chernesky MA, Hook EW 3d, Jones RB, Judson FN, et al. Nonculture tests for genital tract chlamydial infection. What does the package insert mean, and will it mean the same thing tomorrow? Sex Transm Dis 1993;19:243-4.

3 Stamm WE. Laboratory diagnosis of chlamydial infections. In: Bowie WR, Caldwell HD, Jones RP, Mardh P-A, Ridgway GL, Schachter J, et al, eds. Chlamydial infections. Cambridge: Cambridge University Press, 1990 459-70.

4 Pate MS, Hook EW. Laboratory to laboratory variation in Chlamydia trachomatis culture practices. Sex Transm Dis 1995;22:322-6.

5 Stamm WE. Diagnosis of Chlamydia trachomatis genitourinary tract infections. Ann Intern Med 1988;108:710.
6 Taylor-Robinson D. Tests for infection with Chlamydia trachomatis. Int $\mathcal{F}$ STD AIDS. 1996;7:19-26.

7 Clarke LM, Sierra MF, Daidone BJ, Lopez N, Covino JM, McCormack WM. Comparison of the Syva MicroTrac enzyme immunoassay and Gen-Probe PACE 2 with cell culture for diagnosis of cervical Chlamydia trachomatis infection in a high-prevalence female population. $\exists$ Clin Microbiol 1993;31:968-71.

8 Iwen PC, Walker RA, Warren KL, Kelly DM, Hinrichs $\mathrm{SH}$, Linder J. Evaluation of nucleic acid-based test (PACE 2C) for simultaneous detection of Chlamydia trachomatis and Neisseria gonorrhoeae in endocervical speci mens. F Clin Microbiol 1995;33:2587-91.

9 Stary A, Teodorowicz L, Horting-Müller I, Nerad S, Storch $M$. Evaluation of the Gen-Probe PACE 2 and the MicroTrak enzyme immunoassay for diagnosis of Chlamydia trachomatis in urogenital samples. Sex Transm Dis 1994;21:26-30.

10 Schachter J, Stamm WE, Quinn TC, Andrews WW Burczak JD, Lee $\mathrm{HH}$. Ligase chain reaction to deteces Chlamydia trachomatis infection of the cervix. F Clino Microbiol 1994;32:2540-3.

11 Quinn TC, Welsh L, Lentz A, Crotchfelt K, Zenilman J Newhall J, et al. Diagnosis by Amplicor PCR of Chlamydia trachomatis infection in urine samples from women and men attending sexually transmitted disease clinics. F Clin Microbiol 1996;34:1401-6.

12 Ossewaarde JM. New methods in diagnostic and epidemiological research of Chlamydia trachomatis infections: theo tide is turning molecular. F Eur Acad Dermatol Venereol 1995;5:111-23

13 Smith TF, Weed LA. Comparison of urethral swabs, urine and urinary sediment for the isolation of Chlamydia. $\mathcal{F}_{2}$ Clin Microbiol 1975;2:134-5.

14 Stary A, Najim Belinda, Lee HH. Vulval swabs as an alter native specimen for the detection of genital chlamydiat infection in women by ligase chain reaction. F Cline

15 Stary A, Genc M, Heller-Vitouch C, Mardh P-Ao Chlamydial antigen detection in urine samples by immunofluorescence tests. Infection 1992;20.53-6.

16 Leonardi GP, Seitz M, Edstrom R, Cruz J, Costello P Szabo K. Evaluation of three immunoassays for detection of Chlamydia trachomatis in urine specimens from asymp= tomatic males. $\mathcal{f}$ Clin Microbiol 1992;30:2793-6.

17 Ehret JM, Leszcynski JC, Douglas JM, Genova SL Chernesky MA, Moncada J, et al. Evaluation of Chlamydiazyme enzyme immunoassay for detection of Chlamydia trachomatis in urine specimens from men? f Clin Microbiol 1993;31:2702-5.

18 Sanders JW, Hook III EW, Welsh LE, Sepherd ME, Quinn TC. Evaluation of an enzyme immunoassay for detection of Chlamydia trachomatis in urine of asymptomatic men. Clin Microbiol 1994;32:24-7.

19 Stary A, Tomazic-Allen S, Choueiri, Burczak J, Steyrer K, Lee $\mathrm{H}$. Comparison of DNA amplification methods for the detection of Chlamydia trachomatis in first-void urine from asymptomatic military recruits. Sex Transm Dis 1996;23:97-102.

20 Chernesky MA, Jang D, Lee $\mathrm{HH}$, Burczak JD, $\mathrm{Hu} \mathrm{H}_{3}$ Sellors J, et al. Diagnosis of Chlamydia trachomatis infec-es tions in men and women by testing first-void urine by lig=ase chain reaction. 7 Clin Microbiol 1994;32:2682-5.

21 Lee HH, Chernesky MA, Schachter J, Burczak JD Andrews WW, Muldoon S, et al. Diagnosis of Chlamydia trachomatis genitourinary infection in women by ligase chain reaction assay of urine. Lancet 1995;345:213-6.

22 Bassiri M, Hu HY, Domeika MA, Burczak J, Svensson LO Lee $\mathrm{HH}$, et al. Detection of Chlamydia trachomatis in urine specimens from women by ligase chain reaction.
Clin Microbiol 1995;33:898-900.

23 Toye B, Peeling RW, Jessamine P, Claman P, Gemmill I Diagnosis of Chlamydia trachomatis infections in asymp-O tomatic men and women by PCR assay. 7 Clin Microbio h

24 Smith BL, Cummings MC, Benes S, McCormack WMN Evaluation of vaginal tampons for collection of specimens for detection of chlamydial infection. Sex Transm Dise 1996;23:127-30.

25 Stary A, Hartmann B, Schuh E, Kerschbaumer M, Jurecka U. Detection of Chlamydia trachomatis in urine and urogenital specimens using transcription mediated ampli-? fication (Gen-Probe). Third European Chlamydia Meeting, Vienna 1996; p 318.

26 Pasternack R, Vourinen P, Miettinen A. Evaluation of the Gen-Probe Chlamydia trachomatis transcription-mediated amplification assay with urine specimens from women. $\mathcal{f}$ Clin Microbiol 1997;35:676-8. 